\title{
Distribution functions for hard thermal particles in QCD
}

\author{
J Frenkel * \\ Instituto de Física, Universidade de São Paulo, \\ São Paulo, Brazil \\ J C Taylor \\ DAMTP, Centre for Mathematical Sciences, Cambridge University, \\ Cambridge, UK
}

October 30, 2018

\begin{abstract}
We find a closed-form for the distribution function (defined in terms of a Wigner operator) for hot coloured particles in a background gluon field, in the hard thermal loop approximation. We verify that the current is the same as that derived from the known effective action.
\end{abstract}

\section{Introduction}

Thermal QCD is beset with IR divergence difficulties. A necessary first step is to sum the "hard thermal loops" - loop diagrams with external momenta small compared to the temperature $T$ [1]. These hard thermal loops have been summed up to give an effective action [2]. In the hard thermal approximation, the hot particles (quarks or gluons) behave semi-classically; and this suggests the use of a transport equation. Such equations have been written down by treating the hot quanta as particles [3], and also derived by defining the distribution function as the thermal expectation value of a Wigner operator [4].

In the second approach, it has been usual to derive the transport equation from the Wigner operator, and then solve that equation in the hard thermal approximation. The purpose of this paper is to omit the intermediate step (the transport equation), and to find a closed form for the distribution function (that is, the expectation value of the Wigner operator) in the hard thermal approximation. This closed form expression ought to encode the same information as the effective action. We verify this by showing that the current derived from the

*This work was supported in part by CNPq and FAPESP, Brazil 
distribution function is identical to that coming from the functional derivative of the effective action.

In order to illustrate our method, we first treat hot scalar "quarks" in the background colour field. Then we treat spinor quarks. Finally, we discuss the case where the hot particles are gluons, using the background field formalism.

\section{Hot scalar particles}

In order to illustrate our approach as simply as possible, we first treat the unphysical example of a hot scalar field $\phi_{a}$ in the fundamental representation of $S U(n), a=1, \ldots, n$, in a slowly varying background field $A_{\mu, a b}$, where we take the $n \times n$ matrix $A$ to be antihermitean and traceless and to include the coupling $g$ in its definition. We work in Lorentz space, with metric $(+,-,-,-)$.

We define a distribution function to be the thermal expectation value $\langle\ldots$. (with any zero-temperature part subtracted) of a Wigner operator. We use the symbol $W$ to denote this expectation value. Thus we define (we denote hermitean conjugation by ${ }^{*}$ )

$$
\begin{gathered}
W_{a b}(A ; x, k)= \\
\frac{1}{(2 \pi)^{4}} \int d^{4} y \exp (i k . y) U_{a c}\left(A ; x, x_{+}\right)\left\langle\phi_{c}\left(x_{+} ; A\right) \phi_{d}^{*}\left(x_{-} ; A\right)\right\rangle\left[U^{*}\left(A ; x, x_{-}\right)\right]_{d b},
\end{gathered}
$$

where

$$
x_{+}=x+\frac{1}{2} y, \quad x_{-}=x-\frac{1}{2} y,
$$

and

$$
U\left(A ; x, x_{ \pm}\right)=P \exp \left[\int_{0}^{1 / 2} d u y \cdot A(x \pm u y)\right]
$$

and $P$ denotes path-ordering of the matrices $A$. We have put $A$ in the argument of $\phi$ in (1) to emphasize that $\phi$ satisfies the field equation in the background field $A$. It follows from (1) that $W$ is hermitean.

Under a gauge transformation $\Lambda$,

$$
\begin{aligned}
\phi(x) & \rightarrow \Lambda(x)^{-1} \phi(x), \\
A_{\mu}(x) \rightarrow A_{\mu}^{\Lambda} & \equiv \Lambda^{-1} A_{\mu} \Lambda+\Lambda^{-1} \partial_{\mu} \Lambda .
\end{aligned}
$$

Then from (5)

$$
U\left(x, x_{+}\right) \rightarrow \Lambda^{-1}(x) U\left(x, x_{+}\right) \Lambda\left(x_{+}\right) ;
$$

so that, from (1),

$$
W(x) \rightarrow \Lambda^{-1}(x) W(x) \Lambda(x) .
$$

To calculate $W$ in (1), we need solutions of the field equation for $\phi$, in the background field $A$. This equation is

$$
\left[\partial_{\mu}+A_{\mu}\right]^{2} \phi=-m^{2} \phi
$$


(we give the scalar "quarks" a mass $m$, since this costs very little extra complication). We seek an approximate solution of the form

$$
\begin{gathered}
\phi_{a}=(2 \pi)^{-3 / 2} \int d^{4} k \delta^{+}\left(k^{2}-m^{2}\right) \\
\times\left[V_{a b}(A ; x, k) \exp (-i k \cdot x) a_{b}(k)+V_{a b}(A ; x,-k) \exp (i k . x) b_{b}^{*}(k)\right]
\end{gathered}
$$

where $a$ is an annihilation operator and $b^{*}$ is a creation operator (normalized to $\left.\left[a_{a}(\mathbf{k}), a_{b}^{*}\left(\mathbf{k}^{\prime}\right)\right]=2|\mathbf{k}| \delta^{3}\left(\mathbf{k}-\mathbf{k}^{\prime}\right) \delta_{a b}\right)$.

We assume that $k$ is large compared to $A$, and to the rates of variation of $V$ and $A$. Then (9) satisfies (8) provided that

$$
\left[2 i k . \partial+2 i k . A-(\partial+A)^{2}\right] V=0 .
$$

Write

$$
V=V_{0}+V_{1}+\ldots
$$

where (defining the covariant derivative $D$ )

$$
\begin{gathered}
(k . \partial+k . A) V_{0} \equiv k \cdot D V_{0}=0, \\
2 i k . D V_{1} \approx(\partial+A)^{2} V_{0} \equiv D^{2} V_{0} .
\end{gathered}
$$

A unitary solution of (12) is

$$
V_{0}(A ; k, x)=P \exp \left[-\int_{-\infty}^{0} d u k \cdot A(x+u k)\right]=1-(k \cdot \partial)^{-1}(k \cdot A)+\ldots
$$

We assume that $k . \partial$ has a unique inverse when acting on functionals of $A$ (this is discussed in equation (1.7) of [5]); so that we also have

$$
V_{0}(A ; k, x)=\bar{P} \exp \left[\int_{0}^{\infty} d u k \cdot A(x+u k)\right]=\left[V_{0}(A ;-k, x)\right]^{-1} .
$$

From (13)

$$
\begin{gathered}
V_{1} \approx(2 i k . D)^{-1} D^{2} V_{0}, \\
V_{1}^{*} \approx-V_{0}^{*} D^{* 2}\left(2 i k . D^{*}\right)^{-1},
\end{gathered}
$$

where we make the convention that differential operators like $D^{*}$ act to the left.

Using the solutions (9), and taking the thermal expectation value of the creation an annihilation operators, we get from (1) 


$$
\begin{gathered}
W_{a b}(x, k)=\frac{1}{(2 \pi)^{4}} \int d^{4} y e^{i k \cdot y} \int d^{4} k^{\prime} \delta^{+}\left(k^{\prime 2}-m^{2}\right) N\left(\left|k_{0}^{\prime}\right|\right) \\
\left\{e^{-i k^{\prime} \cdot y}\left[U\left(x, x_{+}\right) V\left(x_{+}, k^{\prime}\right) V^{*}\left(x_{-}, k^{\prime}\right) U^{*}\left(x, x_{-}\right)\right]_{a b}+\left(k^{\prime} \rightarrow-k^{\prime}\right)\right\},
\end{gathered}
$$

where $N$ is the Bose function, and the colours of the quarks in the heat bath have been summed over.

Now let $B_{\mu}(A ;, x, k)$ be defined to be the covariant field which coincides with $A$ in the gauge where $k . A=0$; so by definition

$$
k . B(A ; x, k)=0 .
$$

This means that there is a gauge transformation, $V_{0}(A ; x, k)$ such that

$$
V_{0}^{-1} B_{\mu} V_{0}=A_{\mu}^{\left(V_{0}\right)} \equiv V_{0}^{-1} A_{\mu} V_{0}+V_{0}^{-1} \partial_{\mu} V_{0} \equiv V_{0}^{-1} D_{\mu} V_{0} .
$$

From (19), this requires that $V_{0}$ should satisfy (12), which justifies the use of the notation $V_{0}$. B satisfies the equation (using $\mathcal{D}$ defined in (26) below)

$k . \mathcal{D} B_{\mu} \equiv k . \partial B_{\mu}+k . A B_{\mu}-B_{\mu} k . A=k_{\nu} F_{\nu \mu} \equiv k . \partial A_{\mu}-\partial_{\mu} k . A+k . A A_{\mu}-A_{\mu} k . A$.

This is right because it is a covariant equation, and in the gauge where $k \cdot A=0$ it reduces to $k . \partial(B-A)=0$.

In the hard thermal approximation, $k$ is large compared compared to other dimensional quantities. We assume that this means that $y$ in (1) is small compared to $x$.

We will now separate three contributions to $W$. Type (i) is leading order, where we neglect $y$ and $V_{1}$. This term is independent of $A$, and is simply

$$
W_{(i) a b}=\frac{1}{(2 \pi)^{3}} N\left(\left|k_{0}\right|\right) \delta\left(k^{2}\right) \delta_{a b}
$$

as we would expect.

In type (ii), we neglect $V_{1}$ in (11) and work to first order in $y$ in $U$ and $V_{0}$. Type (iii) terms contain $V_{1}$, and for these we can neglect $y$ in $U$ and $V_{0}$.

For the type (ii) term, then, we replace $V$ by $V_{0}$ in (18), and expand to first order in $y$, to get

$W_{(i i)}=\frac{1}{(2 \pi)^{4}} \int d^{4} y e^{i k \cdot y} \int d^{4} k^{\prime} \delta^{+}\left(k^{\prime 2}-m^{2}\right) N\left(\left|k_{0}^{\prime}\right|\right)\left[e^{-i k^{\prime} . y} R\left(x, k^{\prime}, y\right)+\left(k^{\prime} \rightarrow-k^{\prime}\right)\right]$

where

$$
\begin{gathered}
R=\left[y \cdot A(x)+\left((y / 2) \cdot \frac{\partial}{\partial x} V_{0}\right) V_{0}^{*}-V_{0}\left((y / 2) \cdot \frac{\partial}{\partial x} V_{0}^{*}\right)\right] \\
=y \cdot B\left(x, k^{\prime} ; A\right),
\end{gathered}
$$


where we have used (20) together with $V_{0}^{*} V_{0}=1$.

So (23) gives

$$
W_{(i i)}=\frac{-i}{(2 \pi)^{3}} \frac{\partial}{\partial k_{\mu}}\left[\delta\left(k^{2}\right) N\left(\left|k_{0}\right|\right) B_{\mu}(x, k ; A)\right],
$$

where we have used that $B(-k)=B(k)$ from (21).

In what follows, we will now find it useful to define a "2-sided" covariant derivative $\mathcal{D}$, acting on matrices, by

$$
\mathcal{D}_{\mu} X \equiv\left[D_{\mu}, X\right]=\frac{\partial}{\partial x_{\mu}} X+\left[A_{\mu}, X\right] .
$$

If we let $\mathcal{D}$ act on the product $X Y$ of two matrices (with the same argument $x)$, we get from $(26)$

$$
\mathcal{D}_{\mu}[X(x) Y(x)]=\left[D_{\mu} X\right] Y+X\left[Y D_{\mu}^{*}\right],
$$

where we make the convention that the conjugate operator $D^{*}$ acts to the left (as a differential operator), so that

$$
Y \mathcal{D} *_{\mu} \equiv\left[D_{\mu} Y^{*}\right]^{*} .
$$

Note that, in spite of the notation, $\mathcal{D}$ is not an $n \times n$ matrix differential operator acting from the left. Rather it is an $n^{2} \times n^{2}$ matrix differential operator; so that, acting on a matrix function $X$,

$$
\left[\mathcal{D}_{\mu} X\right]_{a b}=\left(\mathcal{D}_{\mu}\right)_{a b, c d} X_{c d}
$$

Note that, from (21),

$$
k \cdot \mathcal{D}\left(B_{\mu}-A_{\mu}\right)=-\partial_{\mu} k \cdot A .
$$

We shall require the inverse of the operator $k . \mathcal{D}$, which we will write shortly as $(k \cdot \mathcal{D})^{-1}$. This is an $n^{2} \times n^{2}$ non-local operator. More explicitly:

$$
k \cdot \mathcal{D}\left\langle x, a b\left|(k . \mathcal{D})^{-1}\right| x^{\prime}, a^{\prime} b^{\prime}\right\rangle=\delta^{4}\left(x-x^{\prime}\right) \delta_{a a^{\prime}} \delta_{b b^{\prime}}
$$

and, for any matrix $X(x)$,

$$
\left[(k \cdot \mathcal{D})^{-1} X\right]_{a b}(x)=\int d^{4} x^{\prime}\left\langle x, a b\left|(k \cdot \mathcal{D})^{-1}\right| x^{\prime}, c d\right\rangle X_{c d}\left(x^{\prime}\right)
$$

Now turn to type (iii) terms. In these, we may set $y=0$ in $U$ and $V_{0}$. So, from (16), (17) and (18), we require $S$ where

$$
2 S\left(k^{\prime}\right)=\left[\left(k^{\prime} \cdot D\right)^{-1} D^{2} V_{0}\right] V_{0}^{*}-V_{0}\left[V_{0}^{*} D^{* 2}\left(k^{\prime} \cdot D^{*}\right)^{-1}\right],
$$

where as usual $D^{*}$ is understood to act to the left. From (27),

$$
2 k \cdot \mathcal{D} S=\left(D^{2} V_{0}\right) V_{0}^{*}-V_{0}\left(V_{0}^{*} D^{* 2}\right)=2 \mathcal{D}_{\mu} Y_{\mu}
$$


where

$$
2 Y_{\mu}=\left(D_{\mu} V_{0}\right) V_{0}^{*}-V_{0}\left(V_{0}^{*} D_{\mu}^{*}\right)=2 B_{\mu},
$$

(using (20)). Hence from (23) and using $S(-k)=S(k)$,

$$
W_{(i i i)}=\frac{-i}{(2 \pi)^{3}}(k \cdot \mathcal{D})^{-1} \mathcal{D} \cdot B \delta\left(k^{2}\right) N\left(k_{0}\right) .
$$

Combining the contribution from (36) with the type (ii) contribution (25), we finally get

$$
W_{(i i)}+W_{(i i i)}=\frac{-i}{(2 \pi)^{3}}\left[\left(\frac{\partial}{\partial k_{\mu}} B_{\mu} N\left(\left|k_{0}\right|\right) \delta\left(k^{2}\right)\right)+(k \cdot \mathcal{D})^{-1} \mathcal{D} \cdot B N \delta\left(k^{2}\right)\right]
$$

This is our result for the distribution function in the hard thermal loop approximation.

The current is

$$
J_{\mu}(x)=g \int d^{4} k k_{\mu} W(x, k)=g \int d^{4} k \theta\left(k_{0}\right) k_{\mu}[W(x, k)-W(x,-k)]
$$

being, like $W$, an $n \times n$ covariant matrix. Inserting (37) and integrating by parts, the temperature-dependent part of the current is

$$
J_{\mu}(x)=\frac{i}{(2 \pi)^{3}} \operatorname{int}^{4} k N\left(\left|k_{0}\right|\right) \delta\left(k^{2}\right)\left[B_{\mu}-k_{\mu}(k \cdot \mathcal{D})^{-1} \mathcal{D} . B\right] .
$$

Note that

$$
\mathcal{D}_{\mu} J^{\mu}=0,
$$

and $J$ is hermitean.

We now claim that this current is the functional derivative

$$
i g \frac{\delta \Gamma}{\delta A^{\mu}(x)}
$$

of an effective action (see equation (29) of[2])

$$
\Gamma=-\frac{1}{2(2 \pi)^{3}} \int d^{4} x \int d^{4} k N\left(\left|k_{0}\right|\right) \delta\left(k^{2}\right) \operatorname{tr}[B(x, k ; A)]^{2} .
$$

To verify this, we work out the variation of (37). From (21), this must have the form

$$
\delta B_{\mu}(x)=\delta A_{\mu}(x)+\left[f_{\mu} k . \delta A\right](x),
$$

where $f$ is some (nonlocal) functional of $A$. Therefore

$$
\delta\left[B^{2}\right]=2\left[B_{\mu}+k_{\mu} B . f\right] \delta A_{\mu} .
$$


But since $B^{2}$ is an invariant, (43) must vanish for an infintesimal gauge transformation $\omega$ (using (5) and the definition (26))

$$
\delta A_{\mu}=\mathcal{D}_{\mu} \omega
$$

so

$$
k \cdot \mathcal{D} B \cdot f=-\mathcal{D} \cdot B
$$

or

$$
B . f=-[k \cdot \mathcal{D}]^{-1} \mathcal{D} . B,
$$

and thus (46) inserted into the variation of (41) yields the same as (39), as claimed. (Note that $\delta\left[\operatorname{tr} B^{2}\right]=-2 B_{a b} \delta B_{a b}$.)

\section{Dirac quarks.}

We now generalize from scalar quarks $\phi$ to Dirac quarks $\psi$. Then (1) becomes

$$
W=\frac{1}{(2 \pi)^{4}} \int d^{4} y e^{i k \cdot y} U\langle\psi \bar{\psi}\rangle U^{*},
$$

where we suppress colour indices and spinor indices ( $W$ is a matrix in each sort of index). Instead of (9), we use

$$
\psi_{\alpha a}=\sum_{r}(2 \pi)^{-3 / 2} \int d^{4} k \delta^{+}\left(k^{2}-m^{2}\right)\left[\exp (-i k \cdot x) V_{a b, \alpha}^{r} a_{b}^{(r)}+\ldots .\right],
$$

where $r$ labels two spin states, $a, b$ are colour indices, $\alpha$ is a Dirac index. We expand

$$
V_{a b, \alpha}^{r}=V_{0 a b} u_{\alpha}^{(r)}+V_{1 a b, \alpha}^{(r)},
$$

where

$$
(\gamma \cdot k-m) u^{(r)}=0 .
$$

Now act on $\psi$ in (48) with

$$
-(i \gamma \cdot D+m)(i \gamma \cdot D-m)=(\gamma \cdot D)^{2}+m^{2}=D^{2}+m^{2}+\frac{1}{2} \gamma_{\mu} \gamma_{\nu} F^{\mu \nu}
$$

and use that $(i \gamma \cdot D-m) \psi=0$. In a similar way to equation (13), we find that (suppressing colour indices and Dirac indices)

$$
2 i k . D V_{1}^{(r)}=\left(D^{2} V_{0}\right)+u^{(r)} \frac{1}{2}\left(F^{\mu \nu} V_{0}\right) \gamma_{\mu} \gamma_{\nu} u^{(r)} .
$$

When we work out $W$, there is a sum over the spin states $r$, and we use

$$
\sum_{r} u^{(r)} \bar{u}^{(r)}=(k \cdot \gamma)+m
$$


All the contributions, except the contribution from the $F$ term in (52), will be like (37) except for an extra factor $k \cdot \gamma+m$.

In the contribution from the $F$ term in (52), because the $V_{0}$ and $V_{0}^{*}$ are not differentiated, we may use $V_{0} V_{0}^{*}=1$. Thus we get (using (21))

$$
2 C \equiv\left[\gamma_{\lambda} \gamma_{\nu}(k \cdot \gamma+m)-(k \cdot \gamma+m) \gamma_{\lambda} \gamma_{\nu}\right] F^{\lambda \nu}=-2 F^{\mu \nu} k_{\mu} \gamma_{\nu}=-2(k \cdot \mathcal{D}) B . \gamma
$$

So finally, instead of (34), we have 


$$
\begin{gathered}
W=\frac{-i}{(2 \pi)^{3}} \frac{\partial}{\partial k_{\mu}}\left[\left(B_{\mu} N\left(\left|k_{0}\right|\right) \delta\left(k^{2}-m^{2}\right)(k \cdot \gamma+m)\right]\right. \\
-\frac{i}{(2 \pi)^{3}}\left[(k \cdot \mathcal{D})^{-1}(\mathcal{D} \cdot B)(k \cdot \gamma+m)-B \cdot \gamma\right] N\left(\left|k_{0}\right|\right) \delta\left(k^{2}-m^{2}\right) \\
=\frac{-i}{(2 \pi)^{3}}(k \cdot \gamma+m)\left[\frac{\partial}{\partial k_{\mu}}\left\{B_{\mu} N\left(\left|k_{0}\right|\right) \delta\left(k^{2}-m^{2}\right)\right\}+(k \cdot \mathcal{D})^{-1} \mathcal{D} \cdot B N\left(\left|k_{0}\right|\right) \delta\left(k^{2}-m^{2}\right)\right] .
\end{gathered}
$$

The current is now given by, in place of (38),

$$
J_{\mu, a b}=g \int d^{4} k \operatorname{tr}\left[\gamma_{\mu} W_{a b}\right],
$$

where the trace is over Dirac indices. Using

$$
\operatorname{tr}\left[(k \cdot \gamma+m) \gamma_{\mu}\right]=2 k_{\mu},
$$

we get for $J$ the same as (39) but with a factor 2 in front (there are 2 spin states).

\section{Hot gluons.}

We now turn to hot quantized gluons in a background classical Yang-Mills field. Call the background field $A$ as in previous sections and the quantized field $\mathcal{A}$, so that the total gluon field is $A+\mathcal{A}$. Now we let the indices $a, b, \ldots$ stand for the adjoint representation. It may be convenient to think of $A_{a b \mu}$ as an $\left(n^{2}-1\right) \times\left(n^{2}-1\right)$ antisymmetric matrix. We also define $a_{a}$ by

$$
\mathcal{A}_{a b}=g f_{a b c} a_{c} .
$$

The Lagrangian for $a$ is

$$
\mathcal{L}=\mathcal{L}_{q u}+\mathcal{L}_{\text {nonqu }}
$$

where

$$
\mathcal{L}_{q u}=-\frac{1}{4}\left[D_{a b \mu} a_{b \nu}-D_{a b \nu} a_{b \mu}\right]^{2}-a_{a \mu} F_{a b}^{\mu \nu} a_{b \nu}
$$

is the part quadratic in $a$ (which is the only part directly relevant to 1-loop digrams), and $\mathcal{L}_{\text {nonqu }}$ is the rest. Both parts of $\mathcal{L}$ are separately invariant under

$$
A \rightarrow \Lambda^{-1}(D \Lambda), \quad \mathcal{A} \rightarrow \Lambda^{-1} \mathcal{A} \Lambda,
$$


where as usual $D=\partial+A$. The invariance (60) will guarantee that the $W$ we derive transforms covariantly as in (6). On the other hand, the complete Lagrangian $\mathcal{L}$ is also invariant under

$$
A \rightarrow \Lambda^{-1} A \Lambda, \quad \mathcal{A} \rightarrow \Lambda^{-1}(D+\mathcal{A}) \Lambda .
$$

This gauge transformation justifies the fixing of the gauge in (58), for instance by adding the gauge-fixing term

$$
-\frac{1}{2}(D . a)^{2}
$$

For the Wigner function, there are two possibilities (of the same structure as (1)):

$$
w_{\mu \nu}=-\frac{1}{2(2 \pi)^{4}} \int d^{4} y \exp (i k \cdot y)\left(U a_{\mu}\right)\left(x_{+}\right)\left(U a_{\nu}\right)\left(x_{-}\right)
$$

and

$$
W_{\mu \nu, \lambda \rho}=\frac{1}{4(2 \pi)^{4}} \lim _{m \rightarrow 0}\left[\frac{1}{m^{2}} \int d^{4} y \exp (i k . y)\left(U f_{\mu \nu}\right)\left(x_{+}\right)\left(U f_{\lambda \rho}\right)\left(x_{-}\right)\right],
$$

were we have suppressed the group indices, and $f$ is defined as

$$
f_{\mu \nu}=\mathcal{D}_{\mu} a_{\nu}-\mathcal{D}_{\nu} a_{\mu} .
$$

(A Wigner function like (63) was intoroduced in [6].) In (64), $m$ is some gluon mass, which is to tend to zero.

Both (63) and (64) are invariant under (60), but (63) has the disadvantage that it breaks invariance under(61) ; so it may depend on the gauge-fixing of $a$. For (64), it is not clear how to carry out the limiting process consistently: it is well known that there is no smooth limit from a massive to a massless vector field alone (that is, without additional scalar particles). For this reason, we abandon $W$ in (64) in this paper.

In calculating the (expectation value of) the Wigner function. we need the spin sum of the polarization vectors $e$ (analagous to (53)). In a "physical gauge", we have

$$
\sum_{r=1,2} e_{\mu}^{(r)} e_{\nu}^{(r)}(k)=-g_{\mu \nu}+\frac{k_{\mu} n_{\nu}+k_{\nu} n_{\mu}}{k \cdot n} \equiv P_{\mu \nu},
$$

where $n$ is an arbitrary vector. If we could use $W$ in (64), dependence on $n$ would disappear. But using $w$ in (63), a dependence on $n$ may remain.

The complete quadratic action (59) plus (62) may be written

$$
-\frac{1}{2}\left(D_{\mu} a_{\nu}\right)^{2}-a_{\mu} F^{\mu \nu} a_{\nu}
$$

The equation of motion is

$$
D^{2} a^{\mu}-2 F^{\mu \nu} a_{\nu}=0 .
$$


Like (49), we seek an approximate solution of (67) of the form

$$
V_{a b, \mu}^{(r)}=V_{0 a b} e_{\mu}^{(r)}+V_{1 a b, \mu}^{(r)}+\ldots
$$

Then, using (68) and steps like (12) and (13),

$$
i 2 k \cdot \mathcal{D} V_{1, \mu}^{(r)}=\left(\mathcal{D}^{2} V_{0}\right) e_{\mu}^{(r)}+2\left(F_{\mu \nu} V_{0}\right) e^{(r) \nu} .
$$

The contributions from the first term $\left(V_{0}\right)$ in $(69)$ and the first term in (70) come out very similar to the scalar and Dirac cases, and give a contribution to $w_{\mu \nu}$ :

$$
\frac{i}{2(2 \pi)^{3}}\left\{\frac{\partial}{\partial k_{\lambda}}\left[B_{\lambda} N \delta\left(k^{2}\right) P_{\mu \nu}\right]+(k \cdot \mathcal{D})^{-1} \mathcal{D} \cdot B N \delta\left(k^{2}\right) P_{\mu \nu}\right\}
$$

where $P_{\mu \nu}$ was defined in (66). The contribution from the "spin term", the second term in $(70)$ is

$$
\frac{i}{2(2 \pi)^{3}} \frac{1}{n . k}(k \cdot \mathcal{D})^{-1}\left[n_{\lambda} F_{\lambda \nu} k_{\mu}+k_{\lambda} F_{\lambda \nu} n_{\mu}\right]+(\mu \leftrightarrow \nu)
$$

Neither (71) nor (72) can have physical significance in general, because of their dependence on the arbitrary vector $n$. However, the trace $w \equiv w_{\mu}^{\mu}$ is independent of $n$ : the trace of (72) is zero and the trace of (71) is similar to (37). We hope that we may use this trace with some confidence. So we take the current to be

$$
j_{\mu}=g \int d^{4} k k_{\mu} w=g \int d^{4} k \theta\left(k_{0}\right) k_{\mu}[w(k)-w(-k)],
$$

and then we get the expected contribution (39) from (71) (the gluon field is selfconjugate, but there are 2 polarization states: the trace of (66) gives $P_{\mu}^{\mu}=-2$ ).

In (73), we may us the property

$$
w(-k)=w^{c c}(k)
$$

where $c c$ denotes complex conjugation. Then all reference to negative values of $k_{0}$ in $w$ is removed from (73). In order to prove (74), note that $i$ appears in (63) and (10) only multiplied by $k$ (since $a$ and $A$ are real). Since $w^{*}=w$, we can alternatively (using (73)) write (74) as

$$
j_{\mu a b}=g \int d^{4} k \theta\left(k_{0}\right) k_{\mu}\left[w_{a b}(k)-w_{b a}(k)\right] .
$$

\section{Conclusion}

The main results of this paper are the three closed expressions for the distribution functions: (37), (55) and (71) together with (72). In the last case, we expect only the trace to have physical significance. The secondary result is the verification that the currents obtained from these distribution functions coincide with the current coming from the known hard thermal effective action. 


\section{Bibliography}

1. E. Braaten and R. D. Pisarski, Nucl. Phys. B337, 509 (1990); Nucl. Phys. B339, 310 (1990)

2. J. Frenkel and J. C. Taylor, Nucl. Phys. B334, 156 (1992)

3. P. F. Kelly, Q. Liu, C. Lucchesi and C. Manuel, Phys. Rev. D50, 4209 (1994); V. P. Nair, Phys. Rev. D48, 3432 (1993); D50, 4201 (1994); J. P. Blaizot and E. Iancu, Nucl. Phys. B417, 608 (1994); B421, 565 (1994)

4. U. Heinz, Ann. Phys. 161, 48 (1985); Ann. Phys. 168, 148 (1986); H-T. Elze and U. Heinz, Phys. Reports, 183, 81 (1989)

5. J. Frenkel and J. C. Taylor, Nucl. Phys. B334, 199 (1990)

6. H-T. Elze, Z. Phys.C47, 647 (1990); F. T. Brandt, Ashok Das, J. Frenkel, S. H. Pereira and J. C. Taylor, Phys. Lett. B577, 76 (2003) 